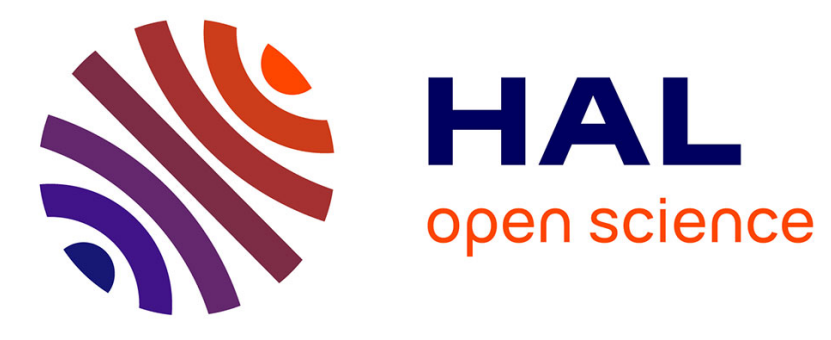

\title{
L'anthropologie dans la formation des enseignants
}

Catherine Choron-Baix

\section{To cite this version:}

Catherine Choron-Baix. L'anthropologie dans la formation des enseignants. Journal des anthropologues, 1990, 42 ("Quelles formations à l'anthropologie? ", Marc-Eric Gruénais et Catherine Quiminal, dir.), pp.49-54. halshs-00010535

\section{HAL Id: halshs-00010535 \\ https://shs.hal.science/halshs-00010535}

Submitted on 11 May 2008

HAL is a multi-disciplinary open access archive for the deposit and dissemination of scientific research documents, whether they are published or not. The documents may come from teaching and research institutions in France or abroad, or from public or private research centers.
L'archive ouverte pluridisciplinaire HAL, est destinée au dépôt et à la diffusion de documents scientifiques de niveau recherche, publiés ou non, émanant des établissements d'enseignement et de recherche français ou étrangers, des laboratoires publics ou privés. 


\section{journal des \\ anthropologues}

\section{2 \\ déc. 1990}

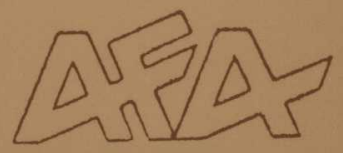




\title{
L'ANTHROPOLOGIE DANS LA FORMATION DES ENSEIGNANTS
}

\author{
Catherine CHORON-BAIX \\ (CEFISEM)
}

L'école connaît aujourd'hui en France d'importantes transformations sociales et culturelles liées à la fréquentation grandissante de populations issues de l'immigration. La coexistence dans l'institution scolaire de valeurs et de comportements hérités de traditions les plus diverses suscite dans la société globale, on le sait, un débat difficile, largement idéologique. Elle pose aussi des questions d'ordre pédagogique particulières.

Elle entraîne notamment une remise en question des contenues et des méthodes d'enseignement en des termes nouveaux. Comment assurer les apprentissages définis par notre société comme fondamentaux (exemples: lecture, écriture, mais aussi activités d'éveil, apprentissages par l'analyse et l'expérimentation) à des enfants dont le milieu familial peut avoir, en matière d'enseignement, d'autres priorités (exemple: accumulation de connaissances apprises par coeur) ? Plus problématique encore, que transmettre, et de quelle manière, à des enfants dits "primoarrivants", qui n'ont aucune connaissance de la langue française mais qui peuvent avoir été scolarisés ailleurs, et possèdent, de ce fait, un certain bagage scolaire dans leur langue maternelle? C'est, pour ces élèves, l'acquisition du français qui devient l'objectif premier, un objectif pour lequel les enseignants ne reçoivent pas de réelle formation.

Tandis que ces difficultés entraînent une véritable mutation des techniques et du métier d'enseignant, la pluralité des cultures présentes dans l'école modifie aussi profondément le vécu en son sein. Maîtres et 
élèves ont à gérer au quotidien un contact pour lequel ils ne sont pas toujours prêts.

Il n'est pas simple pour un enseignant de trouver ses repères dans le tissu complexe des relations qui se nouent entre ses élèves et qui sont parfois la reproduction d'une tradition familiale, elle-même marquée du poids de l'histoire. Par exemple, les enfants asiatiques ne constituent nullement une communauté homogène, mais se partagent au contraire en groupes culturels distincts, chinois, lao, khmer, ou vietnamien, dont les interrelations sont en partie calquées sur le modèle adulte, et perturbées par les traumatismes du passé, et notamment des guerres qui ont secoué l'Asie du Sud-Est ces dernières décennies.

Les relations avec les familles sont elles aussi source d'incompréhension et de malentendus. Que peut répondre une institutrice de maternelle de la banlieue parisienne à une mère d'élève africaine qui lui demande de devenir sa co-épouse? Sans doute les motivations sousjacentes à une telle requête sont-elles claires pour quiconque est familier des cultures d'Afrique et des possibles choix stratégiques d'intégration des populations migrantes. Elles demeurent totalement étranges pour qui en fait la découverte sans aucune préparation.

Dans tous les cas, de telles situations, où l'implication personnelle et la charge émotionnelle sont fortes de part et d'autre, engendrent des réactions mal maîtrisées et souvent inadéquates. Pour y faire face, il faut en avoir une connaissance préalable et disposer d'un certain nombre d'outils d'analyse, parmi lesquels ceux que fournit l'anthropologie sont essentiels.

Ces besoins sont aujourd'hui nettement exprimés par les personnels enseignants, qui sollicitent une information sur les cultures d'origine de leurs élèves étrangers et la recherche de matériaux et de perspectives pédagogiques adaptés à la nouvelle composition socio-culturelle de leurs classes.

Pour répondre à cette demande, le ministère de l'Education nationale a mis en place, en 1981, des structures spécifiques, les CEFISEM, centres de Formation et d'Information sur la scolarisation des enfants de Migrants, dont les fonctions ont été récemment reprécisées (1). 
Instances académiques, constituées de formateurs permanents ayant une expérience de ces questions, ces centres ont pour missions principales d'apporter une aide pédagogique aux personnels des écoles, collèges et lycées, et d'engager auprès de ces derniers des actions de formation sur les spécificités culturelles des enfants d'origine étrangère. Ils ont nécessairement recours aux sciences humaines, et tout particulièrement à l'ethnologie, pour assurer cette tâche.

\section{LA PLACE DE L'ANTHROPOLOGIE MAITRES \\ DANS LA FORMATION DES}

Les données ethnographiques sont en effet la source première d'information concernant les sociétés d'origine des populations immigrées. Dans les stages des CEFISEM, des ethnologues sont régulièrement invités à présenter les résultats de leurs travaux sur une aire culturelle donnée. Dans ces exposés de type monographique, la description des modes d'organisation de la parenté et des rapports sociaux, de la vie matérielle, du système éducatif, du religieux, permet aux auditoires de mesurer la distance qui sépare les comportements appris en famille de ceux qu'inculque l'école française. Dans cette mesure, l'apport ethnologique favorise un rapprochement salutaire des enseignants et de leurs élèves étrangers. Il ne saurait toutefois, ainsi restreint, suffire à une réelle formation en anthropologie, capable de contribuer efficacement à la connaissance du monde de l'école.

Les limites d'une telle approche sont en effet évidentes. A réduire l'ethnologie à l'étude des sociétés lointaines, celles dont sont issus les enfants de migrants, on tend à enfermer ceux-ci dans un ailleurs irréductible, et l'on oublie, du même coup, de s'interroger sur les façons de faire, de penser, de sentir et d'agir de la société globale. C'est pourtant en adoptant pour celle-ci la même démarche et les mêmes principes d'analyse que l'on parvient à croiser les données caractérisant les différents groupes culturels qui la composent, à mieux apercevoir leurs points de contact, leurs ruptures, et les créations culturelles en cours.

D'autre part, en limitant' les connaissances ethnologiques des enseignants aux cultures dites traditionnelles dont seraient porteurs les immigrés, on donne de ceux-ci une image fixe, immuable, qui ne correspond pas à leur réalité. Aucune vague migratoire ne vit la 
transplantation sans se transformer, même si les mutations sont peu perceptibles de l'extérieur. Le travail de l'ethnologue est précisément de mettre en évidence ces changements et d'en trouver les significations, autant de données qui peuvent être utiles aux enseignants, à condition de ne pas être reçues comme des réponses définitives à toute question qui se pose dans leurs classes. En effet, ce que décrit l'ethnologue est une réalité observée à un moment donné, dans un contexte donné, qui ne se répète pas nécessairement à l'identique partout et toujours. Chacune des classes constitue au contraire un milieu unique, où se côtoient des enfants d'horizons divers, créant une sociabilité bien particulière, que l'enseignant doit apprendre à gérer. L'ethnologie peut l'exercer à le faire.

\section{LA NECESSAIRE METHODOLOGIE}

L'une des caractéristiques de l'ethnologie réside en effet dans sa méthodologie, fondée très largement sur un rigoureux travail d'observation à partir duquel peuvent être opérés les rapprochements qui font sens. Cet entraînement au "regard" ethnologique est aujourd'hui l'un des moyens dont doit pouvoir disposer l'enseignant pour mieux maitriser les situations complexes et sans cesse changeantes qui surgissent dans sa classe. Il s'agit pour ce dernier de se maintenir en état d'alerte permanente, prêt à prendre en compte la multiplicité des paramètres, sociaux et culturels qui font la réalité de sa classe: configurations familiales et histoires personnelles des enfants, poids de l'environnement géographique, social et culturel, héritage historique, réflexion sur son propre enracinement culturel et social doivent pouvoir être lus par lui et intégrés dans sa stratégie pédagogique. Pour ce faire, il faut que la formation anthropologique des maîtres comprenne une part d'apprentissage des méthodes.

Instituteurs et professeurs à qui l'on dispense des informations ethnologiques doivent avoir aussi accès à la connaissance des modalités qui permettent de les recueillir. Il leur faut un minimum de formation à l'enquête de terrain. Celle-ci peut aisément leur être proposées dans des domaines qui intéressent au premier chef l'exercice de leur profession. Par exemple, l'exploration d'un quartier, autour d'une école. Menée dans une perspective pluridisciplinaire, où seraient envisagés tour à tour l'étude du bâti et de l'environnement paysager, l'histoire et la composition socio- 
culturelle du peuplement, le complexe commerçant, l'infrastructure économique et socio-culturelle, les formes de la sociabilité, une telle enquête de terrain permettrait aux enseignants d'expérimenter pour leur propre compte le bien-fondé des méthodes de l'ethnologie, et de mieux s'en approprier les résultats.

Le domaine de l'ethnolinguistique fournit un autre champ d'investigation très fécond. Autour du langage s'organisent des systèmes classificatoires, et de multiples savoir et savoir-faire que l'éducateur peut exploiter de multiples manières avec ses élèves dans une dimension comparatiste.

Le contact avec les familles, qui, contrairement à ce qu'on croit, le souhaitent bien souvent sans oser faire les premiers pas, peut être aussi l'occasion d'observer d'autres modes relationnels entre parents et enfants, mari et femme, aînés et cadets.

Ce type d'exercices, dont la liste reste à inventer, doit stimuler l'ouverture à l'autre et la curiosité sans cesse renouvelée, qui sont certainement parmi les meilleures garantes d'un enseignement de qualité.

Si l'on peut se féliciter d'ores et déjà de l'introduction de l'ethnologie dans la formation des maîtres, il faut donc aujourd'hui souhaiter son élargissement.

\section{ELARGIR LA FORMATION ANTHROPOLOGIQUE DES MAITRES}

Il n'est certes pas raisonnable d'envisager que tous les domaines de l'anthropologie soient abordés dans la formation des maîtres. Par contre, il est possible de faire des choix parmi ceux-ci, qui répondent bien aux besoins du métier d'enseignant.

L'anthropologie de l'éducation est de ceux-ci. Apportant une réflexion sur les fonctions des rites de naissance, des stades initiatiques, sur les manières d'apprendre tant dans les sociétés lointaines que dans le monde occidental passé et contemporain, cette partie de la science ethnologique apporterait aux éducateurs une meilleure connaissance et d'eux-mêmes et des autres, et favoriserait peut-être une certaine 
"imagination pédagogique", pour reprendre une expression inspirée de C. Wrigt Mills (2).

L'ethnolinguistique mériterait, elle aussi, une place dans la formation des maîtres. Permettant un travail spécifique sur le langage, elle ouvrirait des perspectives pédagogiques dans des domaines aussi variés que les littératures, orales et écrites, l'ethnoscience, les arts.

L'anthropologie religieuse, quant à elle, apporterait des éléments de connaissance qui manquent cruellement à tous ceux qui ont à travailler et à vivre auprès de populations aux rites éloignés, méconnus, et dangereusement interprétés sans fondement.

Bien sûr, une approche de l'ethnologie du monde moderne, comprenant l'étude des phénomènes migratoires, de la société urbaine et suburbaine, des innovations culturelles nées du contact entre communautés d'origines différentes, devrait figurer aussi au programme.

Ces apports de l'anthropologie, dont on n'a ici que quelques orientations possibles, associés à une initiation méthodologique, deviennent essentiels à la formation des maîtres, parce qu'ils peuvent leur donner un capital de connaissances, de concepts, de savoir-faire qui les rendent plus forts face aux problèmes que soulève l'enseignement aujourd'hui. L'hétérogénéité des populations en présence dans l'école, la confusion échec scolaire-enfants de migrants, le mal de vivre dans ce qu'on appelle parfois le ghetto des structures spécifiques, telles les classes d'initiation, classes d'accueil ou classes de perfectionnement, le flou pédagogique qu'entraînent les transformations de l'école, la culpabilité liée, chez certains, à une mauvaise compréhension de leurs élèves, sont des difficultés quotidiennes que doit affronter l'enseignant. L'éclairage de l'anthropologie, s'il ne résout pas tout, loin s'en faut bien sûr, peut en tous cas lui apporter certains éléments de réponse et des pistes de réflexion. Il augmente aussi ses capacités à évaluer toute situation nouvelle qui se présente à lui et son adaptabilité face à celle-ci. C'est peut-être sur ce dernier point que l'apport de l'ethnologie, parce qu'il est durable, peut être le plus profitable à l'enseignant. 
Numéro épuisé

Fac-similé déposé en Archives ouvertes avec l'autorisation de la revue Scan et reconnaissance de caractères : Eliane Daphy (avril 2006)

Source : exemplaire personnel Eliane Daphy

Ce numéro a été coordonné par Marc-Eric Gruénais et Catherine Quiminal.

La fabrication a été assurée par Béatrice Schatz (Société IODE) et la couverture réalisée par Mbaye Diouf.

Nous tenons à remercier les auteurs des manuscrits qui nous sont envoyés et qui sont autant de contributions à la vie de l'Association.

La forme et le contenu des articles n'engagent que la responsabilité de leurs auteurs. 
Numéro épuisé

Fac-similé déposé en Archives ouvertes avec l'autorisation de la revue Scan et reconnaissance de caractères : Eliane Daphy (avril 2006)

Source : exemplaire personnel Eliane Daphy

\title{
COLLECTIF DE REDACTION
}

\author{
Marie-Christine ANEST -COUFFIN \\ Claude ARDITI \\ JeanCOPANS \\ Rita CORDONNIER \\ Nicole ECHARD \\ Marie·Paule FERRY \\ Jeanine FRIBOURG \\ Marc-Eric GRUENAIS \\ Jean HANNOYER \\ Danièle KINTZ \\ Annie LE PALEC \\ Olivier MASCLET \\ Catherine QUIMINAL \\ Monique SELIM \\ Alex-Louise TESSONNEAU
}

Toute correspondance doit être adressée à :

Journal des anthropologues

A.F.A. - E.H.E.S.S.

1 , rue du 11 Novembre

92120 MONTROUGE

Tél. : (1) 4092 173d

Permanence le mardi après-midi

Voir présentation de la revue et sommaires des numéros sur le site hittp./Www.ala.msh-parts.finjournat.htmit 


\section{SOMMAIRE}

\section{DOSSIER - QUELLES FORMATIONS A L'ANTHROPOLOGIE ?}

Quelles formations à l'anthropologie? M.-E. GRUENAIS et C. QUIMINAL Entretien avec Emmanuel Terray M.-E. GRUENAIS et C. QUIMINAL Formation doctorale d'ethnologie et de sociologie comparative. Louis MALLART-GUIMERA

Le magistère de Sciences sociales de

Paris V - Sorbonne

Dominique DESJEUX et Sophie TAPONIER "Université et musée : une collaboration nécessaire". Le café des ethnologues.

Rencontres étudiantes Daniel TERROLLE Le CNRS et l'anthropologie appliquée : un point de vue Olivier MASCLET L'anthropologie dans la formation des enseignants Gérard LENCLUD Du technicisme à l'accompagnement de dynamiques sociales : la "capitalisation" du G.R.D.R. Notes à propos de l'enseignement de l'anthropologie dans une organisation non gouvernementale médicale Dominique MARTIN

\section{HOMMAGES A MICHEL LEIRIS}

Encens pour Berhane Michel LEIRIS De l'ethnologie considérée comme une tauromachie. Joseph TUBIANA Soixante ans d'amitié Jacques FAUBLEE Michel Leiris devant le colonialisme Claude ARDITI Bibliographie sélective de Michel Leiris Marie-Paule FERRY

\section{ANTHROPOLOGIE VISUELLE}

Société Française d'Anthropologie Visuelle.....

Burkina Faso, en pays lobi, tournage du film "

"Les mémoires de Binduté Da". Michèle FIELOUX

Regards sur les sociétés européennes. Jacques LOMBARD

Séminaires, conférences, festivals 1990 Daniel PELLIGRA Colette PIAULT

\section{NOUVELLES DE LA PROFESSION}

Que viva la reforma! Bertrand GERARD Offres d'emploi La disparition d'une formation en anthropologie Jean-Pierre CASTELAIN 